\title{
Single molecule fluorescence spectroscopy for quantitative biological applications
}

\author{
Ruchuan Liu ${ }^{1}$, Yuliang $\mathrm{Li}^{2, *}$ and Liyu Liu ${ }^{1,2, *}$ \\ 1 College of Physics, Chongqing University, Chongqing 401331, China \\ 2 Key Laboratory of Soft Matter Physics, Institute of Physics, Chinese Academy of Sciences, Beijing 100190, China \\ * Correspondence: Iyliu@cqu.edu.cn
}

Received February 1, 2016; Revised April 9, 2016; Accepted May 9, 2016

\begin{abstract}
Single molecule techniques emerge as powerful and quantitative approaches for scientific investigations in last decades. Among them, single molecule fluorescence spectroscopy (SMFS) is able to non-invasively characterize and track samples at the molecular level. Here, applications of SMFS to fundamental biological questions have been briefly summarized in catalogues of single-molecule counting, distance measurements, force sensors, molecular tracking, and ultrafast dynamics. In these SMFS applications, statistics and physical laws are utilized to quantitatively analyze the behaviors of biomolecules in cellular signaling pathways and the mechanisms of biological functions. This not only deepens our understanding of bio-systems, but also provides a fresh angle to those fundamental questions, leading to a more quantitative thinking in life science.
\end{abstract}

Keywords: single-molecule fluorescence spectroscopy; biomolecule detection; molecular tracking; molecular dynamics; molecular mechanism

\section{INTRODUCTION}

As a non-invasive technique, fluorescence spectroscopy is a favorable characterization tool for researchers and has been applied to various research areas, e.g., physics, chemistry, materials and biology. However, traditional bulk fluorescence measurements only provide average characteristics of samples/molecules and limited spatial resolution, while the detailed information on kinetics, dynamics, and heterogeneous populations is buried. In addition, for heterogeneous and complex systems (especially the biological systems), the tracking of the processive or sequential dynamics and the identification of overlapping transitions and the underlying conformation fluctuations can hardly be achieved by ensemble experiments. In order to overcome these limitations, single molecule approaches by directly monitoring the fluorescence of individual molecules can help to collect data related to individual molecules. Consequently, a clearer picture on the heterogeneous behaviors of the system can be extracted as the entire distributions and time trajectories for target molecules. Therefore, single molecule fluorescence spectroscopy (SMFS) is promising in looking into the details and insights of complex systems at the molecular level, and now becomes a superior approach for biological and medical investigations. In addition, SMFS provides quantitative analysis, which is more and more important in biological studies.

In the past decades, the technology and devices for light detection have been rapidly progressing, and especially the detection sensitivity has reached the single photon level, so the observation and tracking of single molecules by fluorescence have been realized. Recently, the remarkable advancements in SMFS lead to its extensive applications in cutting-edge research areas of physics, chemistry, materials, biology and biotechnology etc. The successful applications include the study of enzymatic dynamics [1-8], rare and transient events $[9,10]$, static and dynamic heterogeneity $[11,12]$, conformation kinetics [13-17] and memory [18], protein folding dynamics [1923], molecular counting and chemometrics [24-28], plasmonic nanostructures [29-31], experimental demonstration of fundamental questions in thermodynamics and statistic mechanics [32-40], and single molecule fluorescence tracking of protein dynamic responses in mammalian cells [41]. Based on the results of these applications, 
the most characteristic property of biomolecules at the molecular scale is that they generally work in stochastic and probabilistic ways in the interplay between thermal fluctuation and molecular interactions. The quantitative data obtained make clear the long time argument that the dynamic and restless interactions of molecules underlie their functions in the cell.

In this review, we will focus on the applications of SMFS to quantitative studies of biological questions, and show its capability as a powerful tool for quantitative biology.

\section{CHARACTERISTICS AND CONDITIONS OF SINGLE MOLECULE FLUORESCENCE}

Compared with that of an ensemble, the fluorescence of a dye molecule (Figure 1A) is many orders of magnitudes weaker in intensity, and is usually recorded as the number of photons emitted. At the same time, the fluorescence of individual molecules is "quantized", i.e., the fluorescence is either on or off, in contrast to the exponential decay of the ensemble samples. In fact, at single-molecule level, reversibly switching between the fluorescent (on) and the dark (off) states, the "blinking" of fluorophores, has been observed. Eventually, the fluorophore goes into the irreversible "photobleaching" and loses the fluorescence at one step (Figure 1B), as a characteristic identification of single molecule fluorescence [42]. Both the fluorescence blinking and the photobleaching have significant impacts on the photostability of the fluorophores and in turn the single-molecule detection of them. Many experimental results shows the contribution of molecular oxygens to the photobleaching, and the triplet state to the blinking of fluorophores: the electron transfers from the excited state to the triplet state at a certain rate and it also takes time to go backwards. Therefore, to lessen the blinking behavior and the elongate the lifetime of fluorophores, a combination of Trolox and enzymatic oxygen-scavenging system has been suggested [43].

Another characteristic property of molecular fluorescence is its polarization. Because most dyes for single molecule fluorescence are organic molecules, and the fluorescence comes from the $\pi$-conjugated function groups, which are usually planar. In ensemble samples, the random orientation of molecules leads to the unpolarized fluorescence. However, at the molecular level, the polarization/anisotropy of the fluorescence can be recorded to track the orientation of individual dyes. Much more information of the rotational property of target molecules can be obtained [44].

Because of the much weaker emission from individual molecules than ensembles, single molecule fluorescence detection usually requires laser excitation, optimized light path with proper dichroic mirrors, filters and other optics,

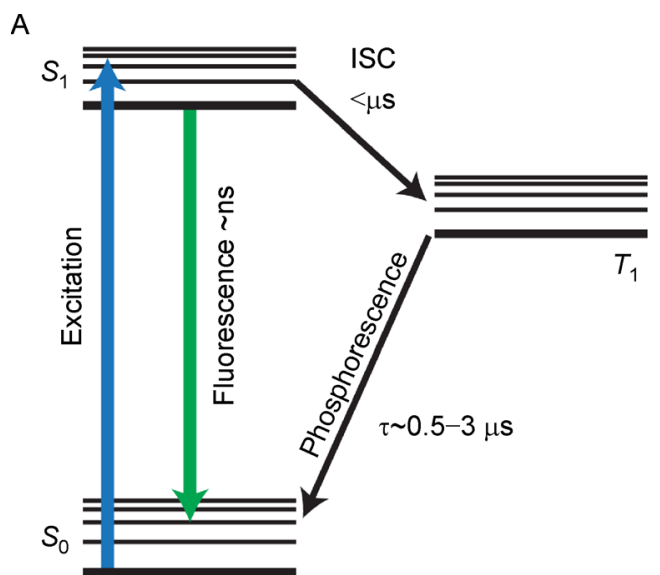

B

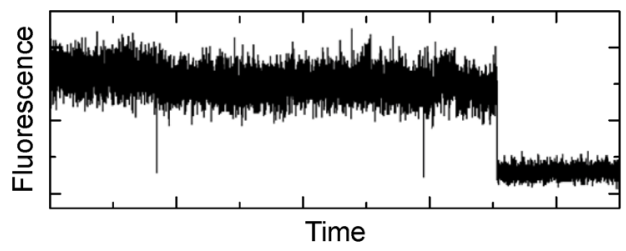

Figure 1. Scheme of Single-molecule fluorescence. (A) An energy level diagram of a fluorescent molecule and (B) a trajectory of a single fluorescent molecule showing the intensity decrement in one step.

as well as the high sensitive detectors, such as avalanche photodiode (APD), photomultiplier tube (PMT) and electron-multiplying charge-coupled device (EMCCD). In addition, due to the particle nature of light, shot noise, which is the square root of the total photon number, is inevitable and contributes to the fluctuation of the fluorescence intensity. In ensemble measurements, shot noise is insignificant as compared with the signal. However, if the background light/fluorescence cannot be reduced to the level of molecular fluorescence, then the weak single molecule fluorescence will be buried in the shot noise of the background. Thus, in order to achieve single-molecule fluorescence detection, special imaging methods are employed to effectively suppress the background lights. Among them, scanning confocal microscopy (SCM) and total internal reflection microscopy (TIRM) are extensively used in SMFS applications, while the principles to realize good signal-to-noise ratios are distinct between these two approaches. In SCM, a laser beam is focused by an objective lens into a small, diffraction limited focal volume, which leads to a much reduced excitation volume; in addition, a beam splitter and a set of filters are applied to pass the fluorescence while blocking the original excitation light, and a pinhole at the back focus plane of the objective lens further blocks scattering light as well as out of focus fluorescence from 
the sample. In TIRM, the evanescent field created by the total reflection of the laser light at the interface can limit the excitation volume to only a couple of hundreds nanometers from the interface, and thus notably reduce the background light; in addition, the laser light path is physically separated from the fluorescence one, blocking most of the scattering of the laser light. Though the SCM and TIRM techniques are also different in other aspects, e.g., imaging speed, 3-D vs. 2-D, both of them have been quite successful in single-molecule fluorescence detections, especially in biological applications.

The fluorescence from individual molecules can be treated as point light sources, but the fluorescence spots have a diffraction limited size according to the Ernst Abbe's equation:

$$
d=\frac{\lambda}{2 n \sin \theta},
$$

where $d$ is the diameter of the spot, $\lambda$ is the fluorescence wavelength, $n$ is the refraction index, $\theta$ is the angle of the focus and $n \sin \theta$ is also called the numerical aperture (NA). Thus, the resolution of fluorescence microscopy is roughly in the order of a couple hundred nanometers. Accordingly, in single-molecule fluorescence experiments, the fluorophores have to be separated at a distance no less than the size of the diffraction limited spot. In other words, the dye molecules has to be diluted enough or at the time frame of imaging only limited number of dye molecules are in the fluorescent state. In case of nonfixed fluorophores, the acquisition time should be short enough to ensure that the displacement of each fluorophore is smaller than the diffraction limited size. On the other hand, due to the limited photons emitted by single fluorophores, the shot noise becomes significant as compared with the fluorescence intensity, the number of photons counted. The signal-to-shot noise ratio can be described as

$$
\frac{\text { Signal }}{\text { Shot-noise }} \propto \sqrt{N}=\sqrt{n_{0} t},
$$

where $N$ is the photon number during the time $t$, and $n_{0}$ is the photon rate, indicating that the shorter the acquisition time for imaging, the worse the signal-to-noise ratio. Accordingly, in tracking single fluorosphore, the time interval of each image should be optimized to balance the signal-to-noise ratio, the spatial resolution, and the temporal resolution.

In the last decade, the fast developing super-resolution microscopy has been proposed to overcome the diffraction limited spatial resolution, including structured illumination microscopy (SIM) [45], stimulated emission depletion microscopy (STED) [46], stochastic optical reconstruction microscopy (STORM) [47], photo-activated localization microscopy (PALM) [48], and other fluorophore localization algorithms, etc. (see reviews on super-resolution imaging in Ref. [49-52]). Briefly, singlemolecule fluorescence can be described by a point-spread function (PSF) centered at the location of fluorophore. Thus, if fluorescent molecules are separated in space or in time, then they can be located by the PSF of singlemolecule fluorescence with accuracy only limited by the molecular fluorescence intensity; or the intensity profile of multiple fluorophores can be deconvoluted as the summary of multiple PSFs using various algorithms. For example, in PALM [48], photo-activable fluorescent proteins are used. At any given moment, only an optically resolvable subset of fluorophores is activated to a fluorescent state, imaged and photobleached. Then other subsets are activated to iterate this process until the localization of numerous fluorophores and the reconstruction of a super-resolution image. Nevertheless, these PSFbased fluorophore localization algorithms can reach a resolution $(\Delta \mathrm{loc})$ defined by the following equation:

$$
\operatorname{loc} \approx \frac{\Delta}{\sqrt{N}},
$$

where $\Delta$ is the half-max width of the PSF, and $N$ the total photons collected from the fluorophore. Presently, a spatial resolution of $20 \sim 30 \mathrm{~nm}$ has been achieved $[41,53]$.

\section{SINGLE MOLECULE COUNTING}

With the capability of detecting individual fluorescent molecules, the most straightforward application is to count the number of available molecules. In biological study, it is essential to tell how many proteins assemble/ bind together at the nanoscale for the understanding of the mechanism of protein functions and the differences between a properly functioning cell and a diseased cell. Thus, it is of great interests to be able to count proteins $[54,55]$ and determine their stoichiometry [56].

To be prepared for single molecule fluorescence microscopy, target proteins and biological molecules are either labelled with fluorescent groups or genetically fused with fluorescent proteins, such as mEos, Dendra, green fluorescent protein (GFP) and mCherry, etc. Especially, the fluorescent proteins are compatible with in vivo experiments and have been widely used in cellular fluorescence images. In addition, evaluations of the photoactivation efficiency have been investigated for quite a few fluorescence proteins as the probes in singlemolecule counting [57,58].

With the available single fluorescence counting, more and more fundamental biological questions are approached either in vitro or in vivo. One of these questions is how the cells "fell" the mechanical stimuli from the environments. It is known that various cells can sense the stiffness of the environment to determine the 
most suitable conditions for them to survive. Most cellular functions are carried out by varied proteins, and cells usually promote certain functions by regulating the expression of corresponding proteins in responding external stimuli. Most of these intracellular processes are biochemical ones, which are different from the external mechanical signals. Therefore, it is of great interest to reveal the mechanotransduction mechanism at the molecular level.

In cell adhesion and focal adhesion, there are quite many proteins, e.g., talin and $\alpha$-catenin, in the chain bridging trans-membrane adhesive proteins (e.g., cadherins and integrins) and cytoskeleton networks. These proteins also bear the forces when cells change the shape in response to external mechanical stimulations. The crystallographic structures of talin and $\alpha$-catenin show the binding sites for vinculin, which is another important protein in the connections between cadherin/integrin and actinin filaments of cytoskeleton. In addition, the binding sites are mostly buried in the helix bundles at the native state of those proteins. Accordingly, the buried binding sites are inaccessible unless the helix bundles are unfolded to expose these sites. On the other hand, mechanical forces are known to unfold proteins, and in many cases the required force can be as low as $\sim 5 \mathrm{pN}[59$ 61]. Therefore, a mechanism can be speculated that forced unfolding of the molecular sensors promotes recruitment of new signaling proteins, which trigger further biochemical cellular pathways.

To identify such molecular mechanotroducting mechanism, the limitation of traditional ensemble approaches has to be overcome. Sheetz et al. combined the single molecule counting and magnetic tweezers to simply count the number of bound vinculins on individual talins under various force conditions (Figure 2) [62]. In their experiments, each talin molecule was chemically bound between a magnetic bead and the substrate, and then incubated in the buffer solution of Alexa-488 labeled vinculin heads. During the incubation, the constant forces were applied to talin molecules through the magnetic beads by an electromagnet. Afterwards, the unreacted vinculin heads were washed away by buffer solutions. Finally, while stretched by the magnetic tweezers, the fluorescence intensity of each talin was monitored over time to catch every single photobleaching event, which corresponds to a vinculin head bound to the talin molecule. As only one talin was expected under a magnetic bead, the number of photobleaching events observed at each fluorescent spot should reflect how many vinculin heads are bound.

The results from the counting experiments (Figure 3) show that the stretching of the talin rods can activate more binding sites for vinculins [62], which are important signaling proteins. In this way, talins function as

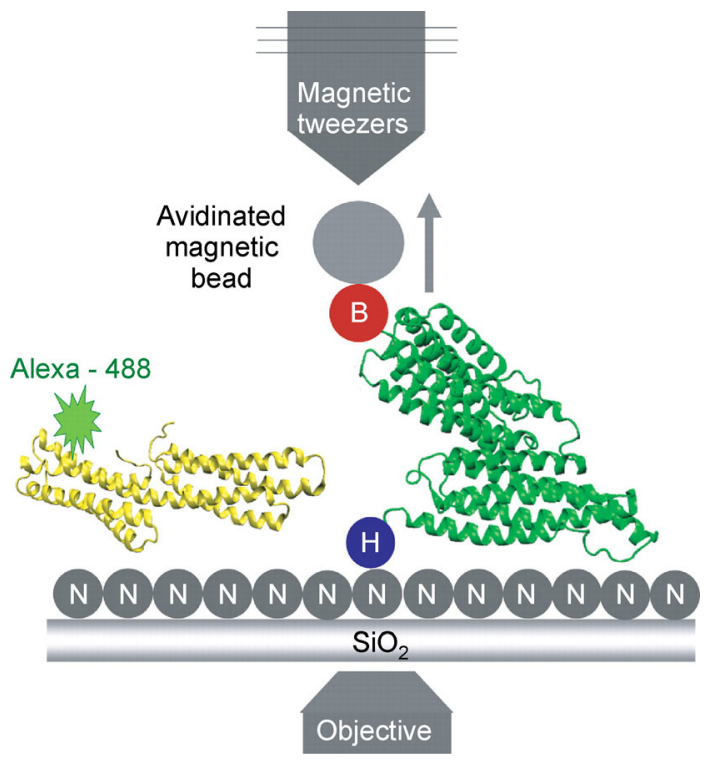

Figure 2. Magnetic tweezers used to measure the binding events. The Ni-NTA (labeled N) grafted slides containing the talin rod fixed through its $6 \times \mathrm{His} \mathrm{N}$ terminus (labeled $\mathrm{H}$ ) to the glass and with the avidinated magnetic bead bound to its biotinylated $C$ terminus (labeled B) was placed over the objective. Alexa 488vinculin head was added to the slides for the period of the incubation. The talin rod and vinculin head structures are represented in green and yellow, respectively. The arrow shows the direction of the movement of the beads when they are pulled using the magnetic tweezers.

mechano-sensor to transduce the mechanical stimuli into biochemical signals. Rececntly, another protein, $\alpha$ catenin at cell adherens junction, was also observed to function in the same way as the mechano-sensing of talin [60]. These experiments indicate that it is a general mechano-transduction mechanism in biological systems to activate the binding of signaling proteins by forced unfolding of mechano-sensing proteins.

Another fundamental biological question is how the expression of a particular gene start and stop. In addition, can any quantitative information be acquired for the transcription and translation of the gene processes? In this aspect, Xie et al. have carried out experiments to look at the expression of individual proteins controlled by a repressed lac promoter in single Escherichia coli cells for the first time [42,63], using a fusion protein consisting a membrane protein, Tsr, and a fluorescent report, Venus. The diffusion of proteins makes it hard to catch the single molecule fluorescence in cytoplasm due to the temporal and spatial resolutions of single-molecule fluorescence techniques. Once the Tsr-Venus molecules attach to $E$. coli's inner membrane and their diffusion is slowed down, which enables single-molecule imaging [42]. 

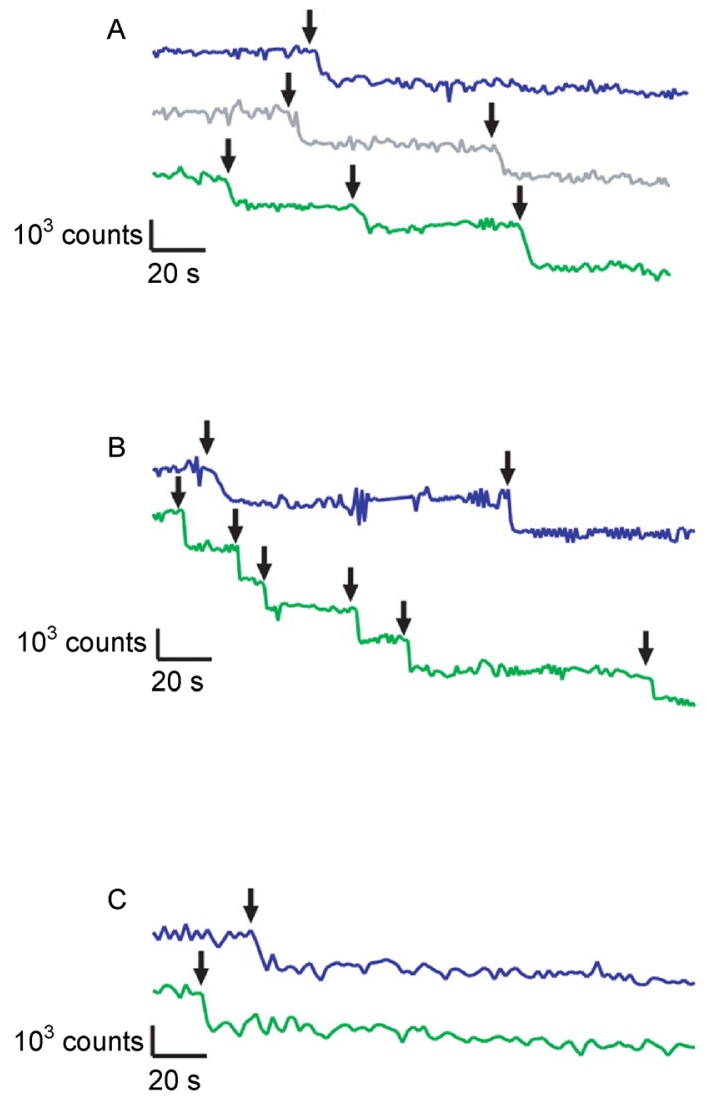
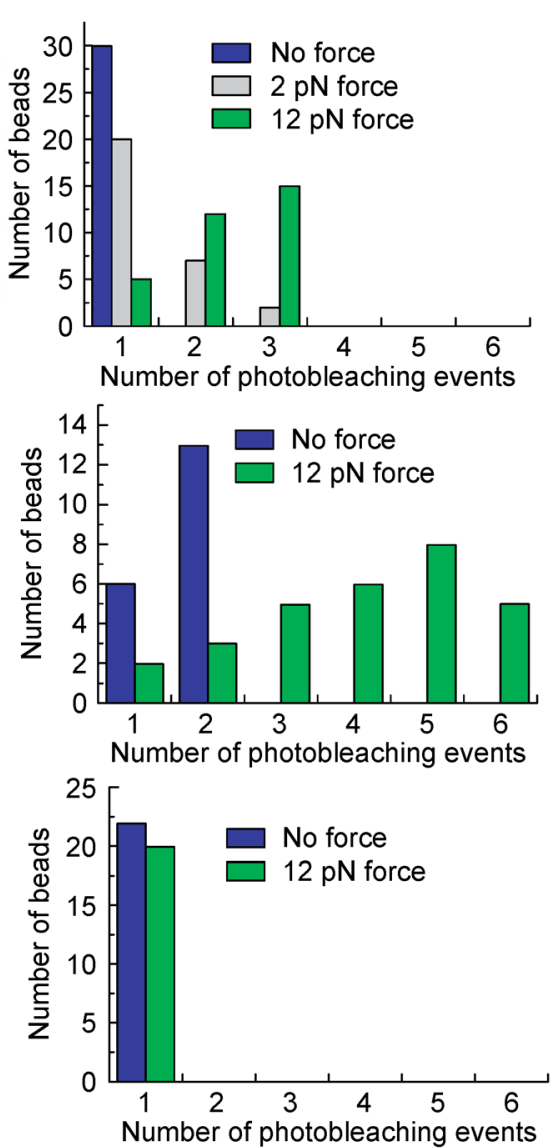

Figure 3. Diagram of photobleaching events of Alexa 488-vinculin head bound to (A) talin rod, (B) dimeric tandem talin rod, and (C) $\alpha$-actinin. Histograms show the number of beads per photobleaching event. In all cases, blue, gray, and green colors correspond with no force, 2-pN force, and 12-pN force applied, respectively. The talin rod, talin dimeric tandem (positive control), and a-actinin (negative control) showed maximally 1 and 3,2 and 6, and 1 and 1 photobleaching events (black arrows) when no force and $12 \mathrm{pN}$ force, respectively, was applied.

In their experiments, diffraction limited spots and onestep photobleaching of each spot were observed, indicating the observation of single fluorescent Tsr-Venus. The expression of Tsr-Venus turned out to be in bursts, and the number of Tsr-Venus and bursts were then counted for cell cycles. The results show that the number of Tsr-Venus in each burst follows the Poisson distribution, suggesting that the gene expression is a stochastic process. Analysis of the average time of cell cycle, the mean value of bursts in each cycle, and the average number of mRNA per cycle and the average lifetime of mRNA implies that under the repression condition only one mRNA is transcribed each time. This indicates the efficiency of the lac repressor: it seldom unbinds the operator region of DNA and quickly rebinds, only allowing transcription initiation of one mRNA molecule. Further counting the number of proteins translated from each mRNA, the distribution follows a single exponential decay. A model of competition between the degradation of mRNA by ribonuclease $\mathrm{E}$ and the translation by ribosomes can be used to quantitatively explain the exponential decay of the production number of proteins per mRNA [42]. Xie et al. have also done a series of experiments to further explorer the dynamics in gene expression in living cells $[64,65]$, and even in human cells [66]. In all these in vivo experiments, the direct monitoring the kinetics of gene expressions has been achieved only by SMFS, and the observation of these fundamental processes in living cells is inapproachable from the ensemble data. The stochastic feature is much more significant from the dynamics at the molecular level. In addition, such a noisy characteristics is only accessible from the singlemolecule approaches, and its origin of the noise in vivo will be further disclosed by the developing singlemolecule approaches, including SMFS. 


\section{SINGLE MOLECULE DISTANCE MEASUREMENTS}

Once target molecules can be identified by their singlemolecule fluorescence, it is straightforward to look at their locations and to measure the distance between molecules. Conventional colocalization use the fluorescence signals to identify whether two molecules of interest are located in the same area $[67,68]$. However, the spatial resolution of the optical microscopy is on the order of a few hundred nanometers, which is much larger than the size of most biological molecules. Thus, it is hard for traditional fluorescence microscopy to answer whether two molecules are really colocalized or to measure their distance by their fluorescence.

Taking advantage of the PSF-based fluorophore localization algorithms, Sheetz et al. further investigated mechanosensing of talin rods in vivo by fusing the $\mathrm{N}$ - and C-terminus of talin with EGFP and mCherry respectively (Figure 4A) [41]. Here, the distance between EGFP and mCherry measures the end-to-end length of the talin, which in its native state is $\sim 51 \mathrm{~nm}$. Using a computational heavily imaging processing method for fluorophore localization, Sheetz et al. were able to measure the distance at a resolution of $\sim 25 \mathrm{~nm}$. By monitoring the changes of the inter-fluorophore distance, the dynamic details of stretching talins were recorded in vivo when cells response to external stimuli.

The results show that the orientation of the talin molecules is strongly correlated to the actin movement and the end-to-end displacement is highly dynamic in vivo. In addition, the displacement is usually significant larger in the peripheral adhesions ( $\sim 100$ to $200 \mathrm{~nm})$ than in the central area. Further experiments were carried out on the displacement after suppressions by Blebbastatin or Y-27632, and dramatic decrement in the displacement is observed as shown in Figure 5A. As previous in vitro single-molecule counting experiments showed that more vinculin heads can bind to the stretched talin rods, cells transiently expressing a vinculin head construct were used to look at the vinculin binding to talin in vivo. A significant change in the length and its dynamics as the result of vinculin binding is observed: a dramatic increase in the displacement to the levels of 400 to $600 \mathrm{~nm}$ and a suppression of the dynamics of length variation were observed, as shown in Figure 5B. These observations can be explained by the strong multiple binding of activated
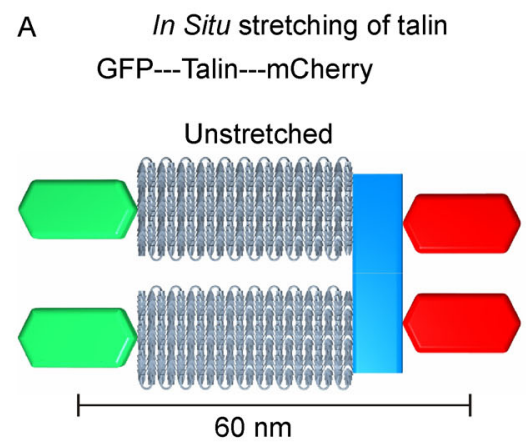
B GFP-Talin-mCherry distributes normally

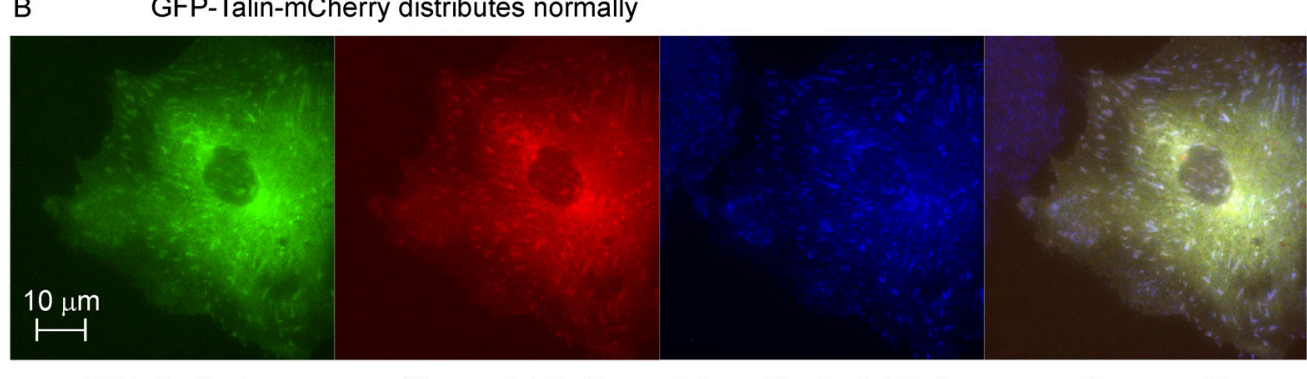

GFP-----T-a-I-i-n-----mCherry

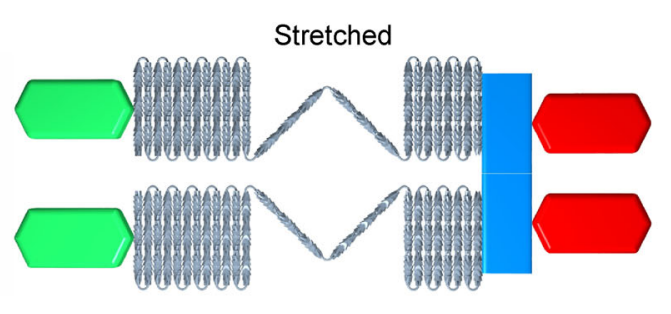

mCherry distribution

Figure 4. EGFP-Talin1-mCherry as a tool to measure talin 1 length. (A) Schematic of stretching of labeled talin molecules: unfolding from a $51 \mathrm{~nm}$ length in the relaxed state. This can be measured in focal adhesions under stretch where the separation of the termini is apparent in two channel TIRF microscopy. Using the same edge detector criterion for both the GFP and mCherry signal reveals the dislocation of the ensemble. (B) Verification that EGFP-Talin1-mCherry distributes as expected for talin. Distribution of EGFP, mCherry, and antibody (alexa 647) staining shows colocalization of the modified and the endogenous talin (green for EGFP, red for mCherry, and blue for alexa 647 in the superposition panel) (this is under conditions of high expression but the same colocalization is observed at low expression). The image was adapted from [41] with permission. 

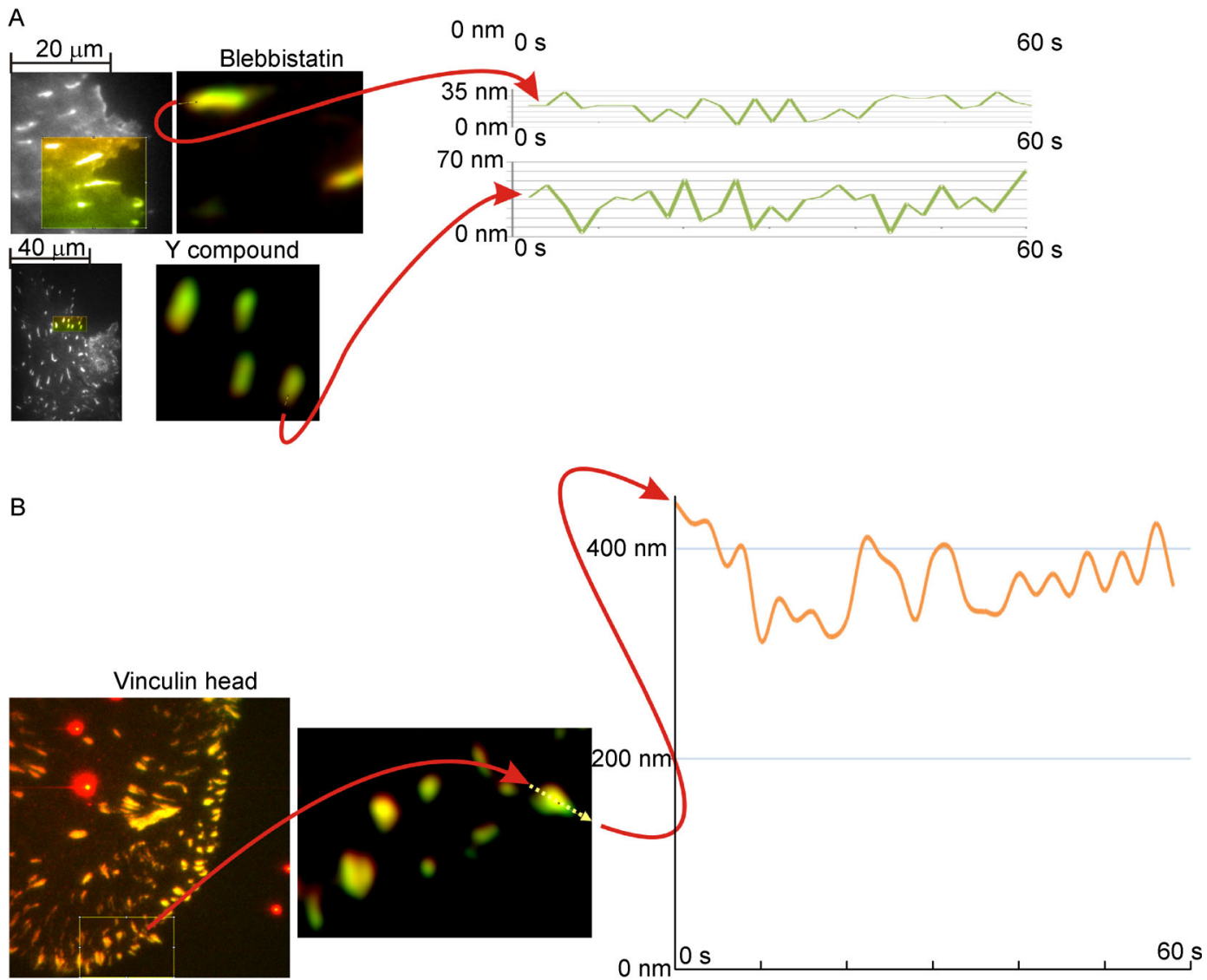

Figure 5. Displacements after suppression. (A) Time evolution of the stretching after adding Blebbastatin or Y-27632, respectively. The total displacement and the amplitude of the stretching are decreased very noticeably. Even though for the Y-27632, it is still clearly larger than the error of observation. (B) Vinculin head added: the vinculin head construct stretched out the talin molecule and left it stretched. There are still observable oscillations in stretching, but the amplitude of change is dramatically suppressed. The image was adapted from [41] with permission.

vinculin head which elongating talin molecules and stabilizing their conformation.

Anyway, the direct measurements of the inter-fluorophore distance is limited by the spatial resolution of the single-molecule fluorescence microscopy, and so far is still beyond $10 \mathrm{~nm}$. To reach below $10 \mathrm{~nm}$, another approach, single-molecule Förster Resonance Energy Transfer (FRET) can be used. FRET refers to the nonradiative energy transfer from an excited fluorophore (donor) to another (acceptor) via dipole-dipole coupling. The FRET efficiency from a donor to an acceptor depends on the distance $(r)$ between them, as given by Equation (4) [44]:

$$
\begin{gathered}
E_{F R E T}=\frac{R_{0}^{6}}{R_{0}^{6}+r^{6}}, \\
R_{0}=8.79 \times 10^{-5} \times\left[n^{-4} \times Q \times \kappa_{2} \times J(\lambda)\right],
\end{gathered}
$$

where $R_{0}$ is the Förster radius, $n$ is the refractive index of the media, $Q$ is the quantum efficiency of the donor in absence of acceptors, $\kappa_{2}$ is the factor describing the relative orientation of the dipoles between donor and acceptor, and $J(\lambda)$ the overlap in their fluorescence spectra [69]. $R_{0}$ is about several nanometers, so FRET is usually observed when the distance between two fluorophores in $1 \sim 10 \mathrm{~nm}$. In turn, single-molecule FRET (smFRET) efficiency can be used as a molecular ruler to measure relative distance with $0.5 \mathrm{~nm}$ resolution over a practical range $<10 \mathrm{~nm}$ [70-73]. Multicolor smFRET allows accurate measurements of the FRET efficiency, following Equation (6) [44]:

$$
E_{F R E T}=\frac{I_{A}}{I_{A}+I_{D} \times \frac{\phi_{A} \times \eta_{A}}{\phi_{D} \times \eta_{D}}},
$$

where $I_{A}$ and $I_{D}$ are the measured fluorescence intensity, $\phi_{A}$ and $\phi_{D}$ are the emission quantum yields, $\eta_{A}$ and $\eta_{D}$ are the detection efficiencies of acceptor (A) and donor 
(D) dyes, respectively.

Due to its capability, the smFRET has been extensively used in biological studies [20,74-79]. Among these applications, Liu et al. [44] used smFRET to investigate the binding kinetics of calmodulin and its target peptide $\mathrm{C} 28 \mathrm{~W}$, as schemed in Figure 6A. In their experiments, calmodulin molecules were labeled with green fluorophore at the N-terminal domain, and the Texas red labeled $\mathrm{C} 28 \mathrm{~W}$ were chosen to bind with calmodulins. Using the Equation (6), the smFRET efficiency was obtained from fluorescence intensities of both fluorophores as measured by a scanning confocal microscope. Accordingly, the relative distance $r / R_{0}$ was calculated by Equation (4). The results are shown in Figure $6 \mathrm{~B}$ and $6 \mathrm{C}$, where both distributions are asymmetric and elongated to one side. If there is only one bound state, then one dominant interfluorophore distance $r$ is expected and the distance distribution should be similar to a symmetric single Gaussian one. The smFRET results evidence that the distribution contains more than one Gaussian component. The authors analyzed the distribution by fitting to two Gaussian peaks with significantly different mean distances (the red and blue curves in Figure 6). The major peak corresponds to a shorter distance between two labels, and attributed to the tightly bound state of the calmodulin/C28W complex. In contrast, the minor peak implies some intermediate state with a larger distance between the N-terminal domain of calmodulin and $\mathrm{C} 28 \mathrm{~W}$. Further measurements on the single-molecule fluorescence polarization also evidenced the conformational fluctuations between bound and loosely bound states in the calmodulin/C28W complex, and probed the timescale in the dynamics. Such fluctuating protein-protein interactions involving bound and loosely bound states are likely general, and have been observed for other cell signaling systems $[80,81]$.

\section{SINGLE-MOLECULE FORCE SENSOR}

The mechanosensing is an essential aspect of cell functions in responding to other partners or extracellular signals, so in order to obtain detailed insight in mechanotransduction, it is important for researchers to measure the molecular forces in vivo, which is expected to be much smaller than those used in AFM unfolding of proteins. In addition, it is almost impossible for present single-molecule techniques, i.e., AFM, optical tweezers and magnetic tweezers, to access target biomolecules in vivo. Therefore, molecular tension sensors are always interesting to researchers.

As discussed above, the smFRET efficiency can be used to measure the extension of proteins, which in turn is a function of the stretching force on the proteins due to their elasticity. Based on this principle, Grashoff et al.
A

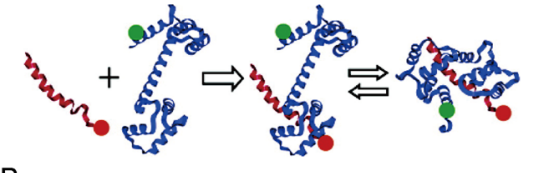

B

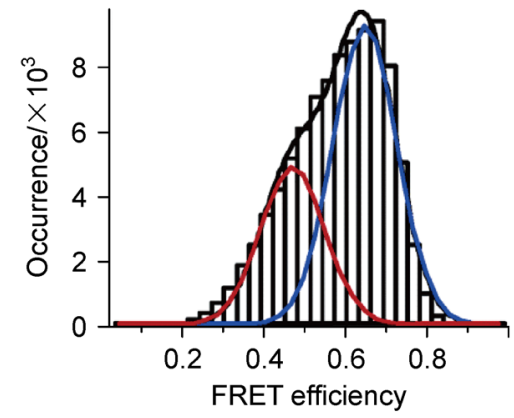

C

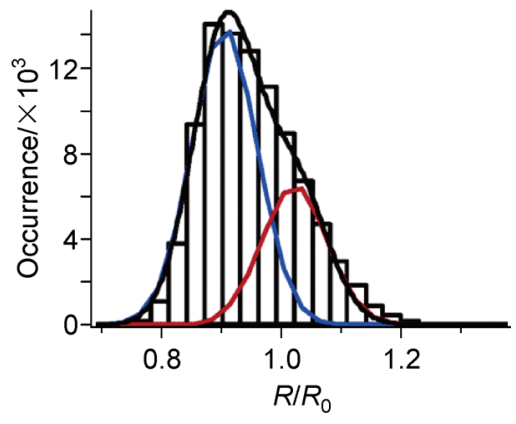

Figure 6. A scheme of two-state interaction model of binding of calmodulin and C28W and singlemolecule FRET results. (A) calmodulin bind with $\mathrm{C} 28 \mathrm{~W}$ in two states: the C-terminal of calmodulin binds to the $\mathrm{N}$-terminal domain of $\mathrm{C} 28 \mathrm{~W}$; calmodulin tightly binds to C28W through both domains. (B) distributions of singlemolecule FRET efficiency; and (C) calculated relative donor-acceptor distance $\left(R / R_{0}\right)$. The blue and red curves are the Gaussian components used to fit the distributions, and the black curve is fitted results. Reprinted with permission from Ref. [43]. Copyright 2006 American Chemical Society.

[61] designed a tension sensor module (TSMod) using a FRET pair, mTFP1 and venus(A20K), which are linked by a 40-amino-acid-long elastic domain (GPGGA) (Figure 7A and 7B). After the FRET efficiency was calibrated as a function of stretching force (Figure 7C) by combining scanning confocal fluorescence microscopy with optical tweezers, the tension sensor was fused with vinculin to calculate forces across vinculin in living cells. An average force in stationary focal adhesions of $\sim 2.5 \mathrm{pN}$ per vinculin was observed. When myosin-dependent contractility was reduced, the FRET index of the sensor increased drastically, indicating the loss of tension across vinculin. By analyzing individual focal adhesions, the FRET index showed that higher tension on vinculin was found within small focal adhesions near protruding edges 
A

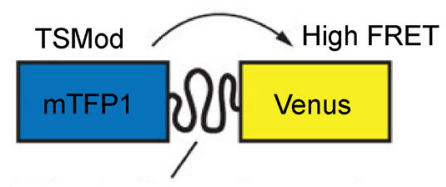

Elastic linker (GPGGA)

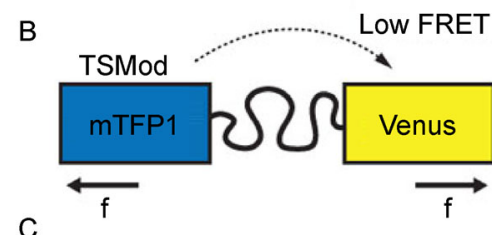

C

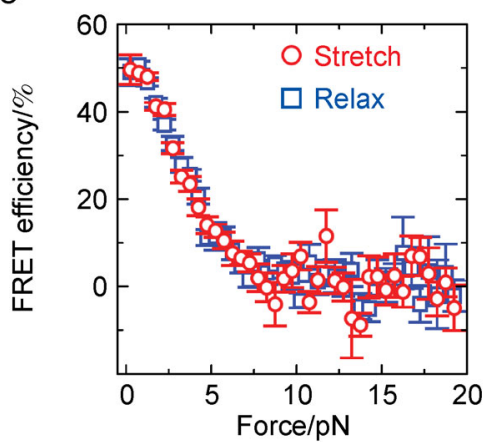

Figure 7. The cartoon of molecular tension sensor and the force calibration. The sensor consists of mTFP1, elastic linker (GPGGA) ${ }_{8}$ and venus $(A)$ without force and $(B)$ once a force is applied the extension of the linker can lower the FRET efficiency. (C) The force is calibrated as a function of FRET efficiency. The image was adapted from [61] with permission.

while lower within large retracting focal adhesions. The time trajectories of FRET index also suggest that the force on vinculin in small focal adhesions at cell edges decreases towards average levels as the focal adhesions enlarge over time.

This smFRET force sensors have already been applied to investigations of molecular forces on cell adhesion proteins E-cadherin [82] and focal adhesion proteins integrin [83]. The results show that E-cadherin is under actomyosin-generated tension no matter whether the cell is in contact with others, while the tension increases at cell-cell contacts under stretching [82]. This also implies that the cadherin/catenin complex may act as an anchor for the cortical actomyosin cytoskeleton. In case of integrin, the results show that the load per integrin on extracellular matrix is in a couple of picoNewtons [83], similar to that in the case of E-cadherin.

\section{SINGLE-MOLECULE TRACKING}

With the capability of counting and localization, single- molecule fluorescence spectroscopy can also be used for single-molecule tracking (SMT), which providing researchers with the unprecedented ability and the ultimate spatial and temporal resolutions to track the behaviors of individual biomolecules, including jostling around, collision, interaction, binding, reaction, dissociation, assembling, etc. In addition to the locations and the movements of single molecules, SMT can also offer the detection of the activation of signaling biomolecules, by in cooperation with other fluorescence techniques, e.g., FRET $[84,85]$. Until now, SMT have been advanced along with the rapid improvement of techniques [86-91] associated with fluorescence microscopy occurring in the fields of physics, chemistry, biology and engineering. A detailed review on the methods and applications of SMT can be found in Ref. [92].

Among those applications, SMT has been successfully applied to an important group of proteins, the processive cytoskeletal motors, including myosin, kinesin, and dynein families. These remarkable molecular motor are able to step over hundreds times successively at speeds of microns per second along microtubule network or actin filaments [93-96], similar to trucks delivering cargos on highways. Though diverse in their structures and biological roles, these motors share the principal characteristics, using the chemical energy (adenosine triphosphate, ATP) to do mechanical work by walking. Properties of these motor molecules, e.g., velocity, processivity and walking mechanism, cannot be readily measured in ensemble assays, while SMT techniques provides the access to investigate these molecular details [97-101]. In all these cases, the hand-over-hand ( $\mathrm{HoH})$ mechanism is demonstrated by statistical analysis of the single-molecule data of step patterns. For example, a distinctly bimodal step size distribution $(74 \mathrm{~nm}$ or $0 \mathrm{~nm}$ ) and bi-exponential decay of the dwell time histogram were observed for myosin $\mathrm{V}$ motors, strongly suggesting the $\mathrm{HoH}$ mechanism other than an inchworm mechanism [98]. In case of kinesin motors, the strictly alternating steps were found and the mechanical strain generated between the two heads acted as the important tension gating in the $\mathrm{HoH}$ walking [102-104]. Besides the processive motors, SMT techniques have also been applied to rotational motors, such ATP synthase [105107] and RNA polymerase [108]. A detailed review on single-molecule application to rotary motors can be found in Ref. [109].

\section{ULTRAFAST DYNAMICS OF SINGLE MOLECULE}

Individual molecule in heterogeneous systems is influenced by the local environment, which causes the variations from molecule to molecule in statics and 
dynamics. Following an individual molecule evolving in time, without synchronizing the ensemble, the dynamics of discrete molecule ranging from femtosecond to nanoseconds can be detected for spontaneous emission, vibrational motions, excitation energy transfer, vibrational relaxation and electronic dephasing, etc. (Figure 8).

The slower processes of a single molecules occurring in nanoseconds have been successfully captured by the conventional single molecular methods from the spontaneous emission of a fluorescence photon, and these have been discussed in the above parts. The faster ones in the femto- to picosecond timescale have not emerged until 2002, when the first observation on excitonic nonlinearity of single GaAs quantum dot with femtosecond resolution at cryogenic temperatures was presented. Following this pioneering work, several time-resolved single molecular spectroscopic techniques have been developed to unravel the electronic and vibrational femtosecond dynamics of individual fluorophores at room temperature. By addressing the issues on electronic coherence, vibrational wavepacket interference, vibrational relaxation decay and coherent electronic energy transfer in light sensitive system (light-harvesting complexes, photo-active proteins and conjugated polymers), the quantum physics being heavily evolved in the molecular systems can be elucidated. However, these tests are unattainable previously in the ensemble measurements, because of the drawback of the ensemble methods in spatial and temporal averaging over an inhomogeneous system. Among these novel results, the observation of coherences in various photosynthetic complexes was an intriguing report. The coherences was already observed during testing the ultrafast energy transfer in photosynthetic complexes before, but it has long been debated and contradictory for the attribution of their origins (electronic or vibrational). For ascertaining the roles and origins of coherence in the efficiency of natural photosynthetic complex, B800-B850 energy transfer in single light harvesting 2 (LH2) complex was detected with a pulseshaped two-color single molecular method [111].

In this experiment, the broad-band 15 fs excitation laser pulse was shaped to be resonant with the absorption frequency of B800 and B850, respectively (Figure 9A), and then the fluorescence from the lowest-energy and emitting B850 exciton state due to energy transfer was detected as shown in Figure 9B. With drawing the

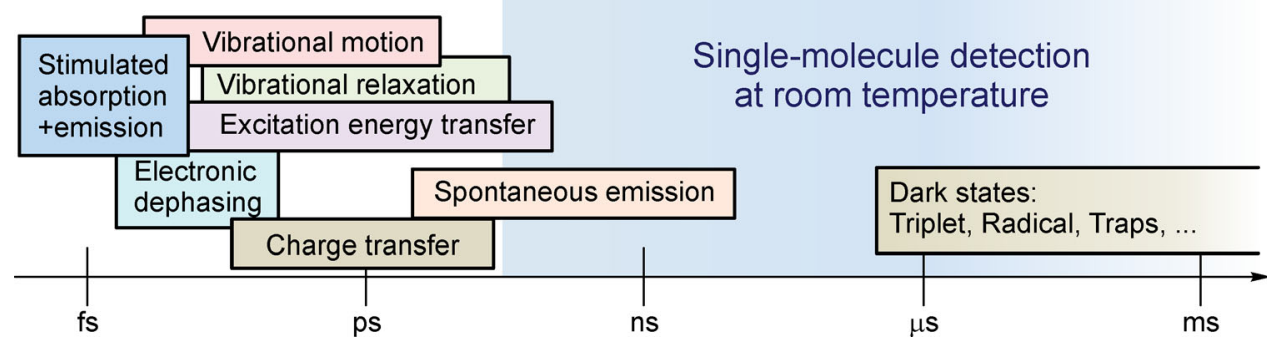

Figure 8. Time schedule of various molecular process (Reproduced from Ref. [110] with permission from The Royal Society of Chemistry).
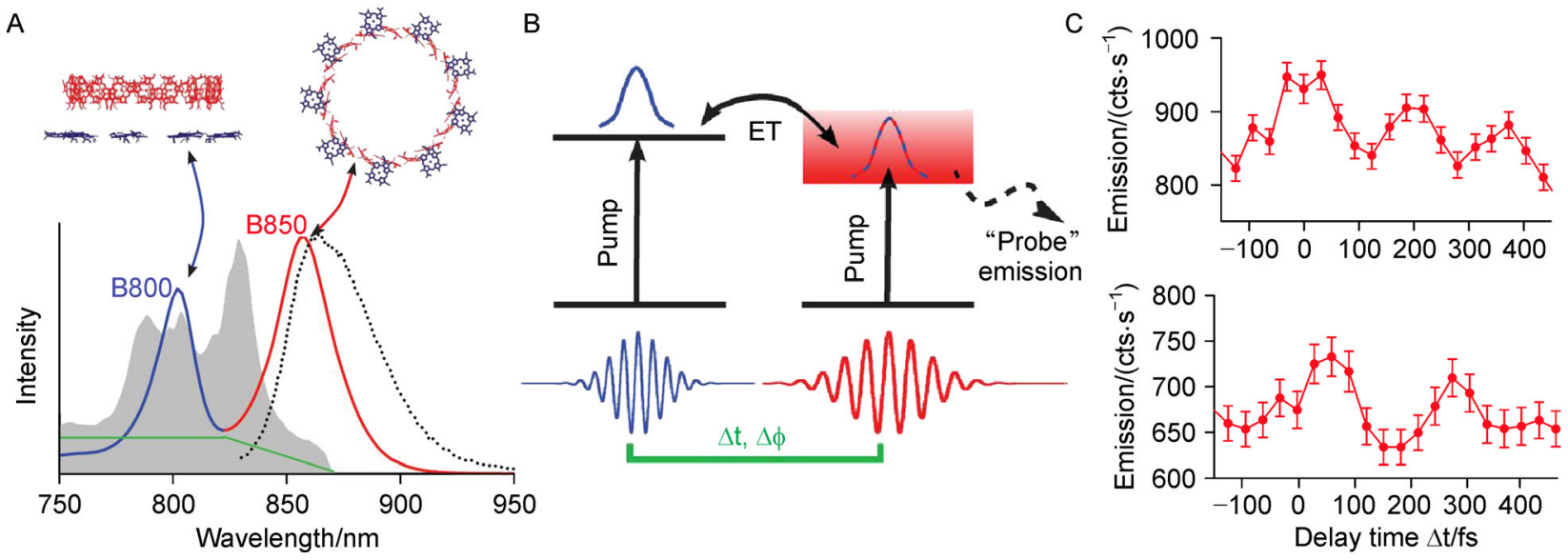

Figure 9. Two color fs phase control of individual LH2 complexes at room temperature (The image was adapted from [112] with permission). 
ultrafast femtosecond single molecular traces from two different individual LH2 complexes, it was found that the fluorescence intensity oscillated up to more than $400 \mathrm{fs}$ with a period of $\sim 200$ fs (Figure 9C), which well matched with the an electronic B800-B850 coupling constant $\mathrm{J}=$ $50 \mathrm{~cm}^{-1}$. However, it cannot be found for the vibrational modes with an energy corresponding to the $200 \mathrm{fs}$ periods in the steady spectroscopic studies. These measurements are different with those from the (much shorter lived) ground-excited state electronic coherence and the vibrational wave packets being created by a specific excitation with an external field. This indicated that the measured oscillations in Figure 9C were definitely caused by the electronic coherences between electronically excited eigenstates of the LH2 complex, and the measured coherence is an intrinsic property of the molecular assemblies, which is important for understanding of the natural molecular systems and their applications[112]. These tests confidently demonstrated that the ultrafast single molecular methods are vital to resolve the dynamics and photophysics of biomolecule complexes which were previously out of reach of physical study.

All schemes presented here are mainly rely on the emitted fluorescence owing to the larger cross section. While most of the interesting systems do not fluoresce, most doped fluorophore can be negative or toxic to the functions of natural (bio-) molecules. Thus it needs efforts for alternative detection schemes such as absorption, stimulated emission, Raman scattering or even enhancement by nano-antennas. Prospectively, the single molecule schedules with femtosecond sensitivity can be ascendant methods for tracking the ultrafast dynamics of individual molecule and the essences of natural (bio-) molecules.

\section{SUMMARY AND OUTLOOK}

In the review, we briefly summarize the single-molecule fluorescence spectroscopic approaches for quantitatively investigation of fundamental biological questions. In these approaches, advanced techniques associated with molecular fluorescence are utilized to record data of individual molecules, and then statistics and physical laws are employed to uncover the quantitative insights of the bio-systems, including location, velocity, force, interaction strength, signaling mechanism, kinetics of processes, ultrafast dynamics, and their evolutions over time. In addition, SMFS measurements can help to identify the minor sub-distributions and transient intermediates, deeper understanding of which is the foundation to the molecular mechanism of bio-systems. These enriched information not only demonstrates and details the existing knowledge and models of the cellular functions, but also provides a fresh angle to these biological questions as the acquirement of the information comes from frontier work in cross disciplinary fields.

Though the fast advances and broad applications have been achieved for SMFS, there are still challenges, especially for applications in quantitative biology:

(i) The cellular processes include both fast and slow processes, so long time tracking will be highly appreciated in biological applications. Consequently, the stability of the fluorophore and microscopic systems are expected to be improved: a more robust fluorophore, or a better system against photobleaching and fluorescence blinking, etc.

(ii) A better spatial and temporal resolution is always the key to uncovering more detailed information from microscopic experiments. The PSF-based localization algorithms are still developing, while fluorophores with a better stability in fluorescence will also improve the resolutions, so enhancements in spectroscopic resolutions can be expected.

(iii) The cellular environment is much crowded by thousands of proteins, and cellular signaling processes involve multiple proteins and their interactions, so multitarget tracking to quantitatively correlate various biomolecules in space and in time sequence is needed and remains as an enormous challenge. The success of Sheetz et al. [41] presents a good start, and further achievements in this regards can be expected.

(iv) Last but not the least, due to the complexity of biological systems, more developments in quantitative methodology for statistical analysis of the SMFS data are of unremitting demands, in order to dig out more insightful understanding of the underline biological functions/mechanism.

Nevertheless, it is expected that the SMFS will become more and more important and irreplaceable in life science, as the progresses of the SMFS and related techniques. In turn, the more and more applications of SMFS will also bring us fresh, better and more enriched details of life.

\section{ACKNOWLEDGEMENTS}

This work was supported by the State Key Development Program for Basic Research of China (No. 2013CB837200), the National Natural Science Foundation of China (Nos. 11474345 and 21573281), the Beijing Natural Science Foundation (No.7154221), and the Fundamental and Advanced Research Program of Chongqing (Grant \# cstc2013jcyjA10047), China. We also gratefully acknowledge support from the Research Start Fund for Talent Recruitment, Chongqing University.

\section{ABBREVIATIONS}

$\begin{array}{ll}\text { SMFS, } & \text { single-molecule fluorescence spectroscopy } \\ \text { APD, } & \text { avalanche photodiode } \\ \text { PMT, } & \text { photomultiplier tube }\end{array}$




$\begin{array}{ll}\text { EMCCD, } & \text { electron-multiplying charge-coupled device } \\ \text { GFP, } & \text { green fluorescent protein } \\ \text { EGFP, } & \text { enhanced green fluorescent protein } \\ \text { NTA, } & \text { Nitrilotriacetic acid } \\ \text { PALM, } & \text { photo-activated localization microscopy } \\ \text { PSF, } & \text { point spread function } \\ \text { E. coli, } & \text { Escherichia coli } \\ \text { FRET, } & \text { Förster Resonance Energy Transfer } \\ \text { smFRET, } & \text { single-molecule FRET } \\ \text { TSMod, } & \text { tension sensor module } \\ \text { mTFP, } & \text { Clavularia teal fluorescent protein } \\ \text { SMT, } & \text { single-molecule tracking } \\ \text { HoH, } & \text { hand-over-hand } \\ \text { LH2, } & \text { light harvesting } 2\end{array}$

\section{COMPLIANCE WITH ETHICS GUIDELINES}

The authors Ruchuan Liu, Yuliang Li and Liyu Liu declare that they have no conflict of interests.

This article does not contain any studies with human or animal subjects performed by any of the authors.

\section{REFERENCES}

1. Hanoian, P., Liu, C. T., Hammes-Schiffer, S. and Benkovic, S. (2015) Perspectives on electrostatics and conformational motions in enzyme catalysis. Acc. Chem. Res., 48, 482-489

2. Liebherr, R. B. and Gorris, H. H. (2014) Enzyme molecules in solitary confinement. Molecules, 19, 14417-14445

3. Janssen, K. P. F., De Cremer, G., Neely, R. K., Kubarev, A. V., Van Loon, J., Martens, J. A., De Vos, D. E., Roeffaers, M. B. and Hofkens, J. (2014) Single molecule methods for the study of catalysis: from enzymes to heterogeneous catalysts. Chem. Soc. Rev., 43, 990-1006

4. Grima, R., Walter, N. G. and Schnell, S. (2014) Single-molecule enzymology à la Michaelis-Menten. FEBS J., 281, 518-530

5. Puchner, E. M. and Gaub, H. E. (2012) Single-molecule mechanoenzymatics. ANN. REV. BIOPHYS., 41, 497-518

6. Xie, S. and Lu, H. P. (1999) Single-molecule enzymology. J. Biol. Chem., 274, 15967-15970

7. Xie, S. (2001) Single-molecule approach to enzymology. Single Mol., $2,229-236$

8. Lu, H. P., Xun, L. and Xie, X. S. (1998) Single-molecule enzymatic dynamics. Science, 282, 1877-1882

9. English, D. S., Furube, A. and Barbara, P. F. (2000) Single-molecule spectroscopy in oxygen-depleted polymer films. Chem. Phys. Lett., $324,15-19$

10. Oukhaled, G., Mathé, J., Biance, A. L., Bacri, L., Betton, J. M., Lairez, D., Pelta, J. and Auvray, L. (2007) Unfolding of proteins and long transient conformations detected by single nanopore recording. Phys. Rev. Lett., 98, 158101

11. Kuzmenkina, E. V., Heyes, C. D. and Nienhaus, G. U. (2005) Singlemolecule Förster resonance energy transfer study of protein dynamics under denaturing conditions. Proc. Natl. Acad. Sci. USA, 102, 1547115476

12. Okumus, B., Wilson, T. J., Lilley, D. M. and Ha, T. (2004) Vesicle encapsulation studies reveal that single molecule ribozyme heterogeneities are intrinsic. Biophys. J., 87, 2798-2806

13. Brucale, M., Schuler, B. and Samorì, B. (2014) Single-molecule studies of intrinsically disordered proteins. Chem. Rev., 114, 32813317

14. Duzdevich, D., Redding, S. and Greene, E. C. (2014) DNA dynamics and single-molecule biology. Chem. Rev., 114, 3072-3086

15. Lu, H. P. (2014) Sizing up single-molecule enzymatic conformational dynamics. Chem. Soc. Rev., 43, 1118-1143

16. Sarkar, S. K., Andoy, N. M., Benítez, J. J., Chen, P. R., Kong, J. S., He, C. and Chen, P. (2007) Engineered holliday junctions as singlemolecule reporters for protein-DNA interactions with application to a MerR-family regulator. J. Am. Chem. Soc., 129, 12461-12467

17. Weiss, S. (2000) Measuring conformational dynamics of biomolecules by single molecule fluorescence spectroscopy. Nat. Struct. Biol., 7, 724-729

18. Pressé, S., Peterson, J., Lee, J., Elms, P., MacCallum, J. L., Marqusee, S., Bustamante, C. and Dill, K. (2014) Single molecule conformational memory extraction: p5ab RNA hairpin. J. Phys. Chem. B, 118, 6597-6603

19. Bavishi, K. and Hatzakis, N. S. (2014) Shedding light on protein folding, structural and functional dynamics by single molecule studies. Molecules, 19, 19407-19434

20. Hofmann, H. (2014) Single-molecule spectroscopy of unfolded proteins and chaperonin action. Biol. Chem., 395, 689-698

21. Lipman, E. A., Schuler, B., Bakajin, O. and Eaton, W. A. (2003) Single-molecule measurement of protein folding kinetics. Science, 301, 1233-1235

22. Schuler, B. and Eaton, W. A. (2008) Protein folding studied by singlemolecule FRET. Curr. Opin. Struct. Biol., 18, 16-26

23. Borgia, A., Williams, P. M. and Clarke, J. (2008) Single-molecule studies of protein folding. Annu. Rev. Biochem., 77, 101-125

24. Kisley, L. and Landes, C. F. (2015) Molecular approaches to chromatography using single molecule spectroscopy. Anal. Chem., $87,83-98$

25. Zhang, H. and Guo, P. (2014) Single molecule photobleaching (SMPB) technology for counting of RNA, DNA, protein and other molecules in nanoparticles and biological complexes by TIRF instrumentation. Methods, 67, 169-176

26. Bharill, S., Fu, Z., Palty, R. and Isacoff, E. Y. (2014) Stoichiometry and specific assembly of Best ion channels. Proc. Natl. Acad. Sci. USA, 111, 6491-6496

27. Arant, R. J. and Ulbrich, M. H. (2014) Deciphering the subunit composition of multimeric proteins by counting photobleaching steps. ChemPhysChem, 15, 600-605

28. Bumb, A., Sarkar, S. K., Wu, X. S., Brechbiel, M. W. and Neuman, K. C. (2011) Quantitative characterization of fluorophores in multicomponent nanoprobes by single-molecule fluorescence. Biomed. Opt. Express, 2, 2761-2769

29. Kneipp, K., Kneipp, H. and Kneipp, J. (2015) Probing plasmonic nanostructures by photons and electrons. Chem. Sci. (Camb.), 6, $2721-2726$

30. Dutta Choudhury, S., Badugu, R. and Lakowicz, J. R. (2015) Directing fluorescence with plasmonic and photonic structures. Acc. Chem. Res., 48, 2171-2180

31. Zohar, N., Chuntonov, L. and Haran, G. (2014) The simplest plasmonic molecules: metal nanoparticle dimers and trimers. J. Photochem. Photobiol. Photochem. Rev., 21, 26-39 
32. Alemany, A., Mossa, A., Junier, I. and Ritort, F. (2012) Experimental free-energy measurements of kinetic molecular states using fluctuation theorems. Nat. Phys., 8, 688-694

33. Collin, D., Ritort, F., Jarzynski, C., Smith, S. B., Tinoco, I. Jr and Bustamante, C. (2005) Verification of the Crooks fluctuation theorem and recovery of RNA folding free energies. Nature, 437, 231-234

34. Gieseler, J., Quidant, R., Dellago, C. and Novotny, L. (2014) Dynamic relaxation of a levitated nanoparticle from a non-equilibrium steady state. Nat. Nanotechnol., 9, 358-364

35. Seifert, U. (2012) Stochastic thermodynamics, fluctuation theorems and molecular machines. Rep. Prog. Phys., 75, 126001

36. Gore, J., Ritort, F. and Bustamante, C. (2003) Bias and error in estimates of equilibrium free-energy differences from nonequilibrium measurements. Proc. Natl. Acad. Sci. USA, 100, 12564-12569

37. Jarzynski, C. (1997) Nonequilibrium equality for free energy differences. Phys. Rev. Lett., 78, 2690-2693

38. Liphardt, J., Dumont, S., Smith, S. B., Tinoco, I. Jr and Bustamante, C. (2002) Equilibrium information from nonequilibrium measurements in an experimental test of Jarzynski's equality. Science, 296, 18321835

39. Wang, G. M., Sevick, E. M., Mittag, E., Searles, D. J. and Evans, D. J. (2002) Experimental demonstration of violations of the second law of thermodynamics for small systems and short time scales. Phys. Rev. Lett., 89, 050601

40. Liphardt, J., Onoa, B., Smith, S. B., Tinoco, I. Jr and Bustamante, C. (2001) Reversible unfolding of single RNA molecules by mechanical force. Science, 292, 733-737

41. Margadant, F., Chew, L. L., Hu, X., Yu, H., Bate, N., Zhang, X. and Sheetz, M. (2011) Mechanotransduction in vivo by repeated talin stretch-relaxation events depends upon vinculin. PLoS Biol., 9, e1001223

42. Yu, J., Xiao, J., Ren, X., Lao, K. and Xie, X. S. (2006) Probing gene expression in live cells, one protein molecule at a time. Science, 311 , 1600-1603

43. Rasnik, I., McKinney, S. A. and Ha, T. (2006) Nonblinking and longlasting single-molecule fluorescence imaging. Nat. Methods, 3, 891893

44. Liu, R., Hu, D., Tan, X. and Lu, H. P. (2006) Revealing two-state protein-protein interactions of calmodulin by single-molecule spectroscopy. J. Am. Chem. Soc., 128, 10034-10042

45. Shao, L., Kner, P., Rego, E. H. and Gustafsson, M. G. (2011) Superresolution 3D microscopy of live whole cells using structured illumination. Nat. Methods, 8, 1044-1046

46. Eggeling, C., Willig, K. I. and Barrantes, F. J. (2013) STED microscopy of living cells - new frontiers in membrane and neurobiology. J. Neurochem., 126, 203-212

47. Rust, M. J., Bates, M. and Zhuang, X. (2006) Sub-diffraction-limit imaging by stochastic optical reconstruction microscopy (STORM). Nat. Methods, 3, 793-796

48. Betzig, E., Patterson, G. H., Sougrat, R., Lindwasser, O. W., Olenych, S., Bonifacino, J. S., Davidson, M. W., Lippincott-Schwartz, J. and Hess, H. F. (2006) Imaging intracellular fluorescent proteins at nanometer resolution. Science, 313, 1642-1645

49. Small, A. and Stahlheber, S. (2014) Fluorophore localization algorithms for super-resolution microscopy. Nat. Methods, 11, 267279

50. Vandenberg, W., Leutenegger, M., Lasser, T., Hofkens, J. and Dedecker, P. (2015) Diffraction-unlimited imaging: from pretty pictures to hard numbers. Cell Tissue Res., 360, 151-178

51. Eggeling, C., Willig, K. I., Sahl, S. J. and Hell, S. W. (2015) Lensbased fluorescence nanoscopy. Q. Rev. Biophys., 48, 178-243

52. Shivanandan, A., Deschout, H., Scarselli, M. and Radenovic, A. (2014) Challenges in quantitative single molecule localization microscopy. FEBS Lett., 588, 3595-3602

53. Horrocks, M. H., Palayret, M., Klenerman, D. and Lee, S. F. (2014) The changing point-spread function: single-molecule-based superresolution imaging. Histochem. Cell Biol., 141, 577-585

54. Jiang, D., Liu, C., Wang, L. and Jiang, W. (2010) Fluorescence singlemolecule counting assays for protein quantification using epifluorescence microscopy with quantum dots labeling. Anal. Chim. Acta, 662, 170-176

55. Földes-Papp, Z. and Baumann, G. (2011) Fluorescence molecule counting for single-molecule studies in crowded environment of living cells without and with broken ergodicity. Curr. Pharm. Biotechnol., $12,824-833$

56. Fricke, F., Beaudouin, J., Eils, R. and Heilemann, M. (2015) One, two or three? Probing the stoichiometry of membrane proteins by singlemolecule localization microscopy. Sci. Rep., 5, 14072

57. Durisic, N., Laparra-Cuervo, L., Sandoval-Álvarez, A., Borbely, J. S. and Lakadamyali, M. (2014) Single-molecule evaluation of fluorescent protein photoactivation efficiency using an in vivo nanotemplate. Nat. Methods, 11, 156-162

58. Lee, S. H., Shin, J. Y., Lee, A. and Bustamante, C. (2012) Counting single photoactivatable fluorescent molecules by photoactivated localization microscopy (PALM). Proc. Natl. Acad. Sci. USA, 109, 17436-17441

59. Lu, C., Wu, F., Qiu, W. and Liu, R. (2013) P130Cas substrate domain is intrinsically disordered as characterized by single-molecule force measurements. Biophys. Chem., 180-181, 37-43

60. Yao, M., Qiu, W., Liu, R., Efremov, A. K., Cong, P., Seddiki, R., Payre, M., Lim, C. T., Ladoux, B., Mège, R. M., et al. (2014) Forcedependent conformational switch of $\alpha$-catenin controls vinculin binding. Nat. Commun., 5, 4525

61. Grashoff, C., Hoffman, B. D., Brenner, M. D., Zhou, R., Parsons, M., Yang, M. T., McLean, M. A., Sligar, S. G., Chen, C. S., Ha, T., et al. (2010) Measuring mechanical tension across vinculin reveals regulation of focal adhesion dynamics. Nature, 466, 263-266

62. del Rio, A., Perez-Jimenez, R., Liu, R., Roca-Cusachs, P., Fernandez, J. M. and Sheetz, M. P. (2009) Stretching single talin rod molecules activates vinculin binding. Science, 323, 638-641

63. Cai, L., Friedman, N. and Xie, X. S. (2006) Stochastic protein expression in individual cells at the single molecule level. Nature, 440 , 358-362

64. Chong, S., Chen, C., Ge, H. and Xie, X. S. (2014) Mechanism of transcriptional bursting in bacteria. Cell, 158, 314-326

65. Elf, J., Li, G.-W. and Xie, X. S. (2007) Probing transcription factor dynamics at the single-molecule level in a living cell. Science, 316, 1191-1194

66. Zong, C., Lu, S., Chapman, A. R. and Xie, X. S. (2012) Genome-wide detection of single-nucleotide and copy-number variations of a single human cell. Science, 338, 1622-1626

67. Jensen, E. (2014) Technical review: colocalization of antibodies using confocal microscopy. Anat. Rec., 297, 183-187

68. Cordelières, F. P. and Bolte, S. (2014) Experimenters' guide to colocalization studies: finding a way through indicators and quantifiers, in practice. Methods Cell Biol., 123, 395-408 
69. Zadran, S., Standley, S., Wong, K., Otiniano, E., Amighi, A. and Baudry, M. (2012) Fluorescence resonance energy transfer (FRET)based biosensors: visualizing cellular dynamics and bioenergetics. Appl. Microbiol. Biotechnol., 96, 895-902

70. Ha, T., Enderle, T., Ogletree, D. F., Chemla, D. S., Selvin, P. R. and Weiss, S. (1996) Probing the interaction between two single molecules: fluorescence resonance energy transfer between a single donor and a single acceptor. Proc. Natl. Acad. Sci. USA, 93, 6264 6268

71. Cornish, P. V. and Ha, T. (2007) A survey of single-molecule techniques in chemical biology. ACS Chem. Biol., 2, 53-61

72. Deniz, A. A., Mukhopadhyay, S. and Lemke, E. A. (2008) Singlemolecule biophysics: at the interface of biology, physics and chemistry. J. R. Soc. Interface, 5, 15-45

73. Slaughter, B. D., Unruh, J. R., Allen, M. W., Bieber Urbauer, R. J. and Johnson, C. K. (2005) Conformational substates of calmodulin revealed by single-pair fluorescence resonance energy transfer: influence of solution conditions and oxidative modification. Biochemistry, 44, 3694-3707

74. Hohng, S. and Ha, T. (2005) Single-molecule quantum-dot fluorescence resonance energy transfer. ChemPhysChem, 6, 956-960

75. Ha, T. (2001) Single-molecule fluorescence resonance energy transfer. Methods, 25, 78-86

76. Hohng, S., Lee, S., Lee, J. and Jo, M. H. (2014) Maximizing information content of single-molecule FRET experiments: multicolor FRET and FRET combined with force or torque. Chem. Soc. Rev., 43, 1007-1013

77. Masson, J.-B., Dionne, P., Salvatico, C., Renner, M., Specht, C. G., Triller, A. and Dahan, M. (2014) Mapping the energy and diffusion landscapes of membrane proteins at the cell surface using high-density single-molecule imaging and Bayesian inference: application to the multiscale dynamics of glycine receptors in the neuronal membrane. Biophys. J., 106, 74-83

78. Mazurkiewicz, J. E., Herrick-Davis, K., Barroso, M., Ulloa-Aguirre, A., Lindau-Shepard, B., Thomas, R. M. and Dias, J. A. (2015) Singlemolecule analyses of fully functional fluorescent protein-tagged follitropin receptor reveal homodimerization and specific heterodimerization with lutropin receptor. Biol. Reprod., 92, 100

79. Jurchenko, C. and Salaita, K. S. (2015) Lighting up the force: investigating mechanisms of mechanotransduction using fluorescent tension probes. Mol. Cell. Biol., 35, 2570-2582

80. Lu, H. P., Iakoucheva, L. M. and Ackerman, E. J. (2001) Singlemolecule conformational dynamics of fluctuating noncovalent DNAprotein interactions in DNA damage recognition. J. Am. Chem. Soc., 123, 9184-9185

81. Tan, X., Nalbant, P., Toutchkine, A., Hu, D., Vorpagel, E. R., Hahn, K. M. and Lu, H. P. (2004) Single-molecule study of protein-protein interaction dynamics in a cell signaling system. J. Phys. Chem. B, 108, 737-744

82. Borghi, N., Sorokina, M., Shcherbakova, O. G., Weis, W. I., Pruitt, B. L., Nelson, W. J. and Dunn, A. R. (2012) E-cadherin is under constitutive actomyosin-generated tension that is increased at cell-cell contacts upon externally applied stretch. Proc. Natl. Acad. Sci. USA, $109,12568-12573$

83. Morimatsu, M., Mekhdjian, A. H., Adhikari, A. S. and Dunn, A. R. (2013) Molecular tension sensors report forces generated by single integrin molecules in living cells. Nano Lett., 13, 3985-3989

84. Suzuki, K. G., Fujiwara, T. K., Sanematsu, F., Iino, R., Edidin, M. and
Kusumi, A. (2007) GPI-anchored receptor clusters transiently recruit Lyn and $G$ alpha for temporary cluster immobilization and Lyn activation: single-molecule tracking study 1. J. Cell Biol., 177, 717730

85. Suzuki, K. G., Fujiwara, T. K., Edidin, M. and Kusumi, A. (2007) Dynamic recruitment of phospholipase $\mathrm{C}$ gamma at transiently immobilized GPI-anchored receptor clusters induces $\mathrm{IP}_{3}-\mathrm{Ca}^{2+}$ signaling: single-molecule tracking study 2. J. Cell Biol., 177, 731742

86. Ha, T. and Tinnefeld, P. (2012) Photophysics of fluorescent probes for single-molecule biophysics and super-resolution imaging. Annu. Rev. Phys. Chem., 63, 595-617

87. Tokunaga, M., Imamoto, N. and Sakata-Sogawa, K. (2008) Highly inclined thin illumination enables clear single-molecule imaging in cells. Nat. Methods, 5, 159-161

88. Nishimura, H., Ritchie, K., Kasai, R. S., Goto, M., Morone, N., Sugimura, H., Tanaka, K., Sase, I., Yoshimura, A., Nakano, Y., et al. (2013) Biocompatible fluorescent silicon nanocrystals for singlemolecule tracking and fluorescence imaging. J. Cell Biol., 202, 967983

89. Kusumi, A., Suzuki, K. G., Kasai, R. S., Ritchie, K. and Fujiwara, T. K. (2011) Hierarchical mesoscale domain organization of the plasma membrane. Trends Biochem. Sci., 36, 604-615

90. Zhang, J., Campbell, R. E., Ting, A. Y. and Tsien, R. Y. (2002) Creating new fluorescent probes for cell biology. Nat. Rev. Mol. Cell Biol., 3, 906-918

91. Zheng, Q., Juette, M. F., Jockusch, S., Wasserman, M. R., Zhou, Z., Altman, R. B. and Blanchard, S. C. (2014) Ultra-stable organic fluorophores for single-molecule research. Chem. Soc. Rev., 43, 1044-1056

92. Kusumi, A., Tsunoyama, T. A., Hirosawa, K. M., Kasai, R. S. and Fujiwara, T. K. (2014) Tracking single molecules at work in living cells. Nat. Chem. Biol., 10, 524-532

93. Huxley, A. F. (2000) Mechanics and models of the myosin motor. Philos. Trans. R. Soc. Lond. B Biol. Sci., 355, 433-440

94. Svoboda, K., Schmidt, C. F., Schnapp, B. J. and Block, S. M. (1993) Direct observation of kinesin stepping by optical trapping interferometry. Nature, 365, 721-727

95. Block, S. M., Goldstein, L. S. and Schnapp, B. J. (1990) Bead movement by single kinesin molecules studied with optical tweezers Nature, 348, 348-352

96. Yanagida, T., Esaki, S., Iwane, A. H., Inoue, Y., Ishijima, A., Kitamura, K., Tanaka, H. and Tokunaga, M. (2000) Single-motor mechanics and models of the myosin motor. Philos. Trans. R. Soc. Lond. B Biol. Sci., 355, 441-447

97. Vale, R. D., Funatsu, T., Pierce, D. W., Romberg, L., Harada, Y. and Yanagida, T. (1996) Direct observation of single kinesin molecules moving along microtubules. Nature, 380, 451-453

98. Yildiz, A., Forkey, J. N., McKinney, S. A., Ha, T., Goldman, Y. E. and Selvin, P. R. (2003) Myosin V walks hand-over-hand: single fluorophore imaging with $1.5-\mathrm{nm}$ localization. Science, 300, 20612065

99. Yildiz, A., Tomishige, M., Vale, R. D. and Selvin, P. R. (2004) Kinesin walks hand-over-hand. Science, 303, 676-678

100. Yildiz, A., Park, H., Safer, D., Yang, Z., Chen, L. Q., Selvin, P. R. and Sweeney, H. L. (2004) Myosin VI steps via a hand-over-hand mechanism with its lever arm undergoing fluctuations when attached to actin. J. Biol. Chem., 279, 37223-37226 
101. Ökten, Z., Churchman, L. S., Rock, R. S. and Spudich, J. A. (2004) Myosin VI walks hand-over-hand along actin. Nat. Struct. Mol. Biol., $11,884-887$

102. Alonso, M. C., Drummond, D. R., Kain, S., Hoeng, J., Amos, L. and Cross, R. A. (2007) An ATP gate controls tubulin binding by the tethered head of kinesin-1. Science, 316, 120-123

103. Block, S. M. (2007) Kinesin motor mechanics: binding, stepping, tracking, gating, and limping. Biophys. J., 92, 2986-2995

104. Yildiz, A., Tomishige, M., Gennerich, A. and Vale, R. D. (2008) Intramolecular strain coordinates kinesin stepping behavior along microtubules. Cell, 134, 1030-1041

105. Sielaff, H. and Borsch, M. (2013) Twisting and subunit rotation in single $\mathrm{F}_{0} \mathrm{~F}_{1}$-ATP synthase. Phil. Trans. R. Soc. B 368, 20120024

106. Tang, G. Q., Roy, R., Ha, T. and Patel, S. S. (2008) Transcription initiation in a single-subunit RNA polymerase proceeds through DNA scrunching and rotation of the N-terminal subdomains. Mol. Cell, 30, 567-577

107. Börsch, M., Diez, M., Zimmermann, B., Reuter, R. and Gräber, P. (2002) Stepwise rotation of the gamma-subunit of $\mathrm{EF}_{0} \mathrm{~F}_{1}$-ATP synthase observed by intramolecular single-molecule fluorescence resonance energy transfer. FEBS Lett., 527, 147-152

108. Harada, Y., Ohara, O., Takatsuki, A., Itoh, H., Shimamoto, N. and Kinosita, K. Jr. (2001) Direct observation of DNA rotation during transcription by Escherichia coli RNA polymerase. Nature, 409, 113115

109. Pilizota, T., Sowa, Y. and Berry, R. M. (2009) Single-Molecule Studies of Rotary Molecular Motors. In Handbook of Single-Molecule Biophysics, P. Hinterdorfer and A. Oijen, Editors. PP. 183-216. Publisher: Springer US

110. Brinks, D., Hildner, R., van Dijk, E. M., Stefani, F. D., Nieder, J. B., Hernando, J. and van Hulst, N. F. (2014) Ultrafast dynamics of single molecules. Chem. Soc. Rev., 43, 2476-2491

111. Hildner, R., Brinks, D., Nieder, J. B., Cogdell, R. J. and van Hulst, N. F. (2013) Quantum coherent energy transfer over varying pathways in single light-harvesting complexes. Science, 340, 1448-1451

112. Gösch, M. and Rigler, R. (2005) Fluorescence correlation spectroscopy of molecular motions and kinetics. Adv. Drug Deliv. Rev., 57, $169-190$ 